\title{
Photochemical Degradation of Phenols in Water Using Flavin-Based Photosensitizers
}

\author{
Quan Wang \\ Central South University, Changsha 410000, China
}

\begin{abstract}
Both flavin derivatives were successfully synthesized and characterized by NMR (nuclear magnetic resonance) and mass spectrometry. A slightly modified method for 10-ethyl flavin was developed. Both HPLC (high performance liquid chromatography) and GC-MS data suggested that all phenols were degraded in the presence of either flavin at micromolar concentrations under direct sun light. A rapid breakdown of the phenols with $35-80 \%$ degradation over the course of $2 \mathrm{~h}$ was observed. The degradation efficiency was clearly dependent on the phenol type. The rank order for the degradation efficiency over $2 \mathrm{~h}$ was 4 -chlorophenol and 4-methoxyphenol $(\sim 80 \%)>$ phenoxyacetic acids $(60-65 \%)>$ nitrophenol and phenol $(\sim 35 \%)$.
\end{abstract}

Key words: Riboflavin, phenol, organic synthesis, photosensitizer, photocatalysis.

\section{Introduction}

Water pollution is one of the major environmental problems in many developing countries. Pesticides and herbicides are exemplary pollutants at significant concentrations in water, many of which contain phenols. Once they are up taken by human, phenols are metabolized to generate harmful phenoxy radicals and quinone-type molecules that lead to oxidative stress. Photochemical degradation of phenols is a promising method to remove them from an aquatic environment. In this study, we have synthesized two flavin-based photosensitizers and studied their ability to degrade various phenols present in water using HPLC (high performance liquid chromatography) and GC mass spectrometry.

\section{Types of Flavin-Based Photosensitizers and Phenols}

In the experiment, the synthetic target products are 10-ethyl flavin and 5-ethyl riboflavin, which are derivatives of riboflavin. There is previous research on the photocatalytic degradation of phenols in waste water by vitamin $B_{2}$ and its derivatives. The phenolic

Corresponding author: Quan Wang, master, research fields: environmental chemistry. pollutants in natural water can be degraded easier and faster by exposing to the natural light. In addition, vitamin $B_{2}$ itself is also easy to be decomposed by photooxidation under the intense light [1]. So the experiment principle is to synthesize the specific groups of riboflavin, to see if synthetic riboflavin derivatives could play a similar or better role in this photocatalytic reaction for the degradation of phenols in wastewater.

\subsection{Vitamin $\mathrm{B}_{2}$ and Its Derivatives}

Vitamin $\mathrm{B}_{2}$, also known as riboflavin, the molecular structure as shown in Fig. 1, is a cofactor of the flavoenzymes in human body. And flavoenzymes plays a critical role in the biological oxidation and reduction for the delivery of hydrogen. As the 1,5 of nitrogen in vitamin $B_{2}$ molecule are in the presence of active conjugated double bonds, we determine it can be used as hydrogen donor, also as hydrogen carriers. The biochemical reactions it attends mainly are for transferring iron or providing energy for the reaction of respiratory chain, such as lipid oxidation, aromatic hydroxylation and protein synthesis. Riboflavin as a water soluble vitamin contains a large number of flavoenzymes, which has two derivatives named FAD 


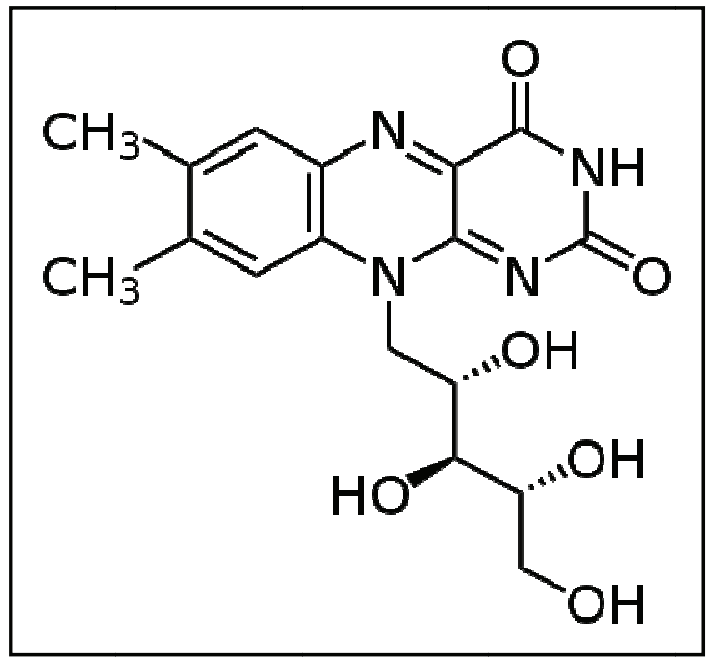

Fig. 1 Molecular structure of Riboflavin.

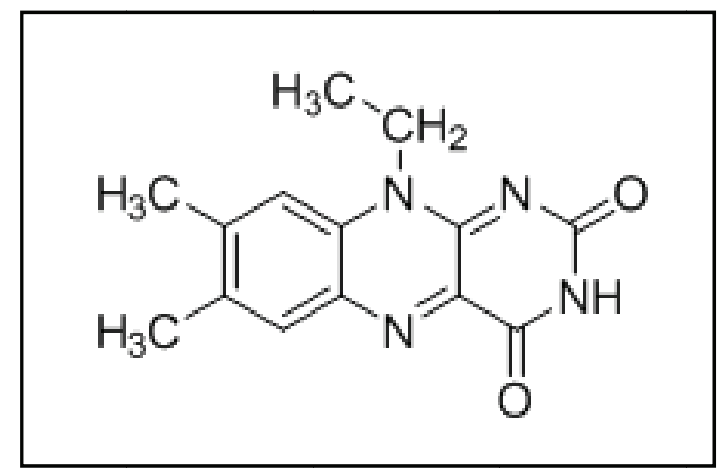

Fig. 2 Molecular structure of 10-ethyl flavin.

(flavin adenine dinucleotide) and FMN (flavin mononucleotide) [2]. They both can be used as an auxiliary factor in a variety of chemical reactions for the electron transferring as a coenzyme. A variety of studies showed that in the aerobic conditions of natural water riboflavin can catalyze the photosensitive oxidation for $\mathrm{p}$-substituted phenols as a photographic sensitizer [3]. Therefore, the experiment in this study synthesized two kinds of derivatives of riboflavin named 10-ethyl flavin and 5-ethyl riboflavin, the molecular structure of 10- ethyl flavin is shown in Fig. 2.

\subsection{The Type of Phenolic Pollutants}

Phenolic pollutants in the process of agricultural production are produced mainly from the overuse of pesticides and herbicide. Its natural biodegradation mechanism is not clear, and the degradation process is slow. There is damage to the ecological environment if they concentrated in natural water. The low concentration phenolic compounds in human body will cause chronic poisoning and higher concentration as reaction with protein, and high concentration will lead to acute poisoning and death.

There are many kinds of phenolic pollutants in water, such as phenol, cresol, nitrophenol and chlorophenol. The compounds used for analysis were phenol, p-nitrophenol, 2,4-dichlorophenoxyacetic acid, 4-chlorophenol, 4-methoxyphenol, and 4-chloro-2-methylphenoxyacetic acid. The first four classes belong to the common phenolic pollutants, and the last two are derivatives of phenolic pollutants presented in the residual of overused pesticides and herbicide. The most representative is phenol, commonly used as raw material for organic synthesis, which is the highest phenolic content types of pollutants in the production and application of pesticides, fertilizers and herbicide. Phenol is soluble in organic solvent and slightly soluble in water. Chlorophenols, chemical formula: $\mathrm{ClC}_{6} \mathrm{H}_{4} \mathrm{OH}$, are often used in pesticides, dyes and other organic synthesis of raw materials or intermediates. [4] Nitrophenol, chemical formula: $\mathrm{NO}_{2} \mathrm{C}_{6} \mathrm{H}_{4} \mathrm{OH}$, is a light yellow crystal, generally used for intermediates in organic synthesis industry. Cresol is colorless or yellowish brown liquid, and similar to phenol in odor and toxicity. It will be darkened when exposed to natural light irradiation. In summary, the overall properties of phenolic compounds are easy to react with aromatic hydroxyl and easy to be replaced. In addition, phenols are easily oxidized, and photo catalytic degradation. The intermediate products in reaction are complex, but they all can be degraded into two carbon dioxide and water in ideal react condition.

\section{Synthetic Derivatives of Riboflavin Phenol for Degradation of Phenols}

\subsection{Synthetic Methods for 10-Ethyl Flavin}

The synthetic method of 10-ethyl flavin is 
expounded. 10-ethyl flavin and 5-ethyl riboflavin were finally used for dealing with 6 kinds of phenols mentioned before to compare the respective treatment effect. 10-ethyl flavin is a yellow solid, in a single synthesis experiment, 20 working days were needed to synthesize a mass of $2 \mathrm{~g} 10$-ethyl flavin. The main reagents and organic solvent for this synthetic experiment are respectively shown in Tables 1 and 2 . 10-ethyl flavin organic synthetic experiment study is divided into 5 steps, and the synthetic route, reagents and intermediates are shown in Fig. 3.

As shown in Fig. 3, the first step in the multistep synthesis of 10-ethyl flavin is the addition of a triflouroacetyl group to an amine 1 as a protecting group. This prevents diakylation of Compound 1. The alkylation of triflouroacetanilide produced from the previous step to prepare Compound 3 was achieved using ethyl iodide as an alkylating agent. The formation of compound 3 was confirmed by mass spectrometry. The third step in the multistep synthesis of 10-ethyl flavin involved the removal of the protecting group to produce Compound 4, which needs to be purified by brief column chromatography. Once our sample reached the bottom of the column, the flowing sample was collected in $10 \mathrm{~mL}$ samples and each tested through TLC (thin layer chromatography) for purity. This was done until the obtained samples showed impurities and then they were combined with the pure orange crystalline solid compound 4 . The fourth step involves the reduction of the nitro group in tin(II) chloride and ethanol followed by basifying the reaction mixture with $\mathrm{NaOH}$. Compound 5 is a small liquid oily substance, and we proceed the next step by adding the reagents to the same flask due to its instability. Compound 5 will react with alloxan monohydrate in the presence of acetic acid and boric acid. After about 5 hours of running the reaction, the solvent is evaporated, filtered

Table 1 Reagents for the synthesis of 10-ethyl flavin.

\begin{tabular}{|c|c|c|c|}
\hline Chemical name & Chemical formula & Supplier & CAS Number \\
\hline 4,5-Dimethyl-2-nitroanaline & $\mathrm{C}_{8} \mathrm{H}_{10} \mathrm{~N}_{2} \mathrm{O}_{2}$ & Sigma-Aldrich & $6972-71-0$ \\
\hline Trifluoroacetic acid anhydride & $\left(\mathrm{CF}_{3} \mathrm{CO}\right)_{2} \mathrm{O}$ & Alfa Aesar & $407-25-0$ \\
\hline Ethyl iodide & $\mathrm{CH}_{3} \mathrm{CH}_{2} \mathrm{I}$ & Alfa Aesar & $75-03-6$ \\
\hline Alloxan monohydrate & $\mathrm{C}_{4} \mathrm{H}_{2} \mathrm{~N}_{2} \mathrm{O}_{4}$ & Alfa Aesar & $2244-11-3$ \\
\hline Sodium bicarbonate & $\mathrm{NaHCO}_{3}$ & Fisher Scientific & $144-55-8$ \\
\hline Sodium chloride & $\mathrm{NaCl}$ & Spectrum Inc & $7647-14-5$ \\
\hline Potassium carbonate & $\mathrm{K}_{2} \mathrm{CO}_{3}$ & Amresco & $584-08-7$ \\
\hline Sodium hydroxide & $\mathrm{NaOH}$ & Fisher Scientific & $1310-73-2$ \\
\hline Tin(II) chloride & $\mathrm{SnCl}_{2}$ & Alfa Aesar & $7772-99-8$ \\
\hline Acetic acid & $\mathrm{CH}_{3} \mathrm{COOH}$ & Fisher Scientific & $64-19-7$ \\
\hline Boric acid & $\mathrm{H}_{3} \mathrm{BO}_{3}$ & Alfa Aesar & $10043-35-3$ \\
\hline
\end{tabular}

Table 2 Organic solvents for the synthesis of 10-ethyl flavin.

\begin{tabular}{llll}
\hline Chemical name & Chemical formula & Supplier & CAS Number \\
\hline Dichloromethane & $\mathrm{CH}_{2} \mathrm{Cl}_{2}$ & EMD & $75-09-2$ \\
Acetonitrile & $\mathrm{CH}_{3} \mathrm{CN}$ & Alfa Aesar & $75-05-8$ \\
Chloroform & $\mathrm{CHCl}_{3}$ & VWR & $67-66-3$ \\
Methanol & $\mathrm{CH}_{3} \mathrm{OH}$ & Alfa Aesar & $67-55-1$ \\
Ethanol & $\mathrm{CH}_{3} \mathrm{CH}_{2} \mathrm{OH}$ & EMD & $64-17-5$ \\
Diethyl ether & $\left(\mathrm{CH}_{3} \mathrm{CH}_{2}\right)_{2} \mathrm{O}$ & EMD & $60-29-7$ \\
Toluene & $\mathrm{C}_{6} \mathrm{H}_{5} \mathrm{CH}_{3}$ & Scholar Chemistry & $108-88-3$ \\
Ethyl acetate & $\mathrm{C}_{4} \mathrm{H}_{8} \mathrm{O}$ & Spectrum Inc & $141-78-6$ \\
Cyclohexane & $\mathrm{C}_{6} \mathrm{H}_{12}$ & Alfa Aesar & $110-82-7$ \\
Ethane & $\mathrm{C}_{2} \mathrm{H}_{6}$ & Alfa Aesar & $74-84-0$ \\
\hline
\end{tabular}




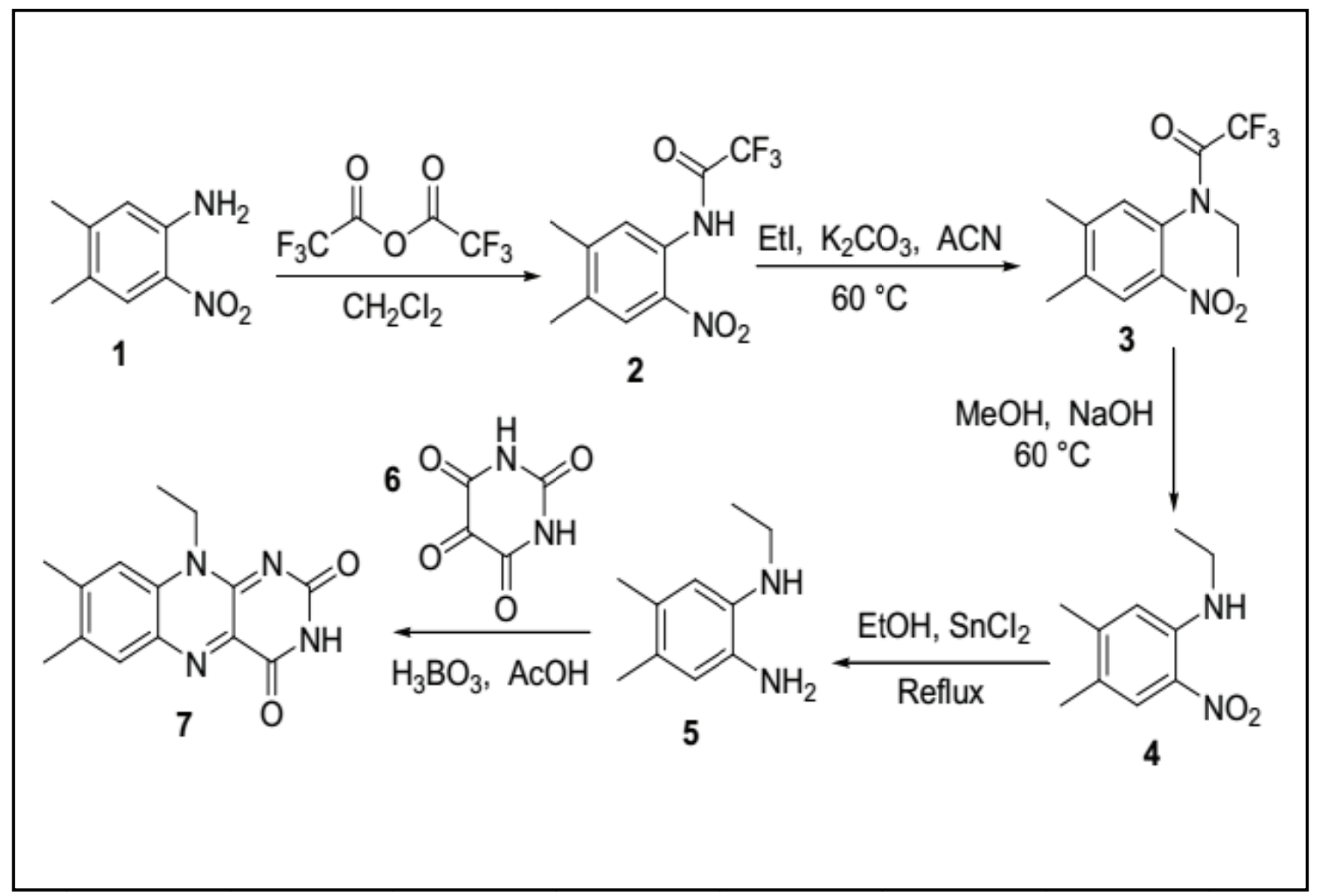

Fig. 3 Synthetic route for 10-ethyl flavin.

(1) 4,5-dimethyl-2-nitroanaline; (2) 4,5-Dimethyl-2-nitrofluoroacetanilide; (3) Compound 3; (4) Compound 4; (5) Compound 5; (6) alloxan monohydrate; (7) 10-ethyl flavin.
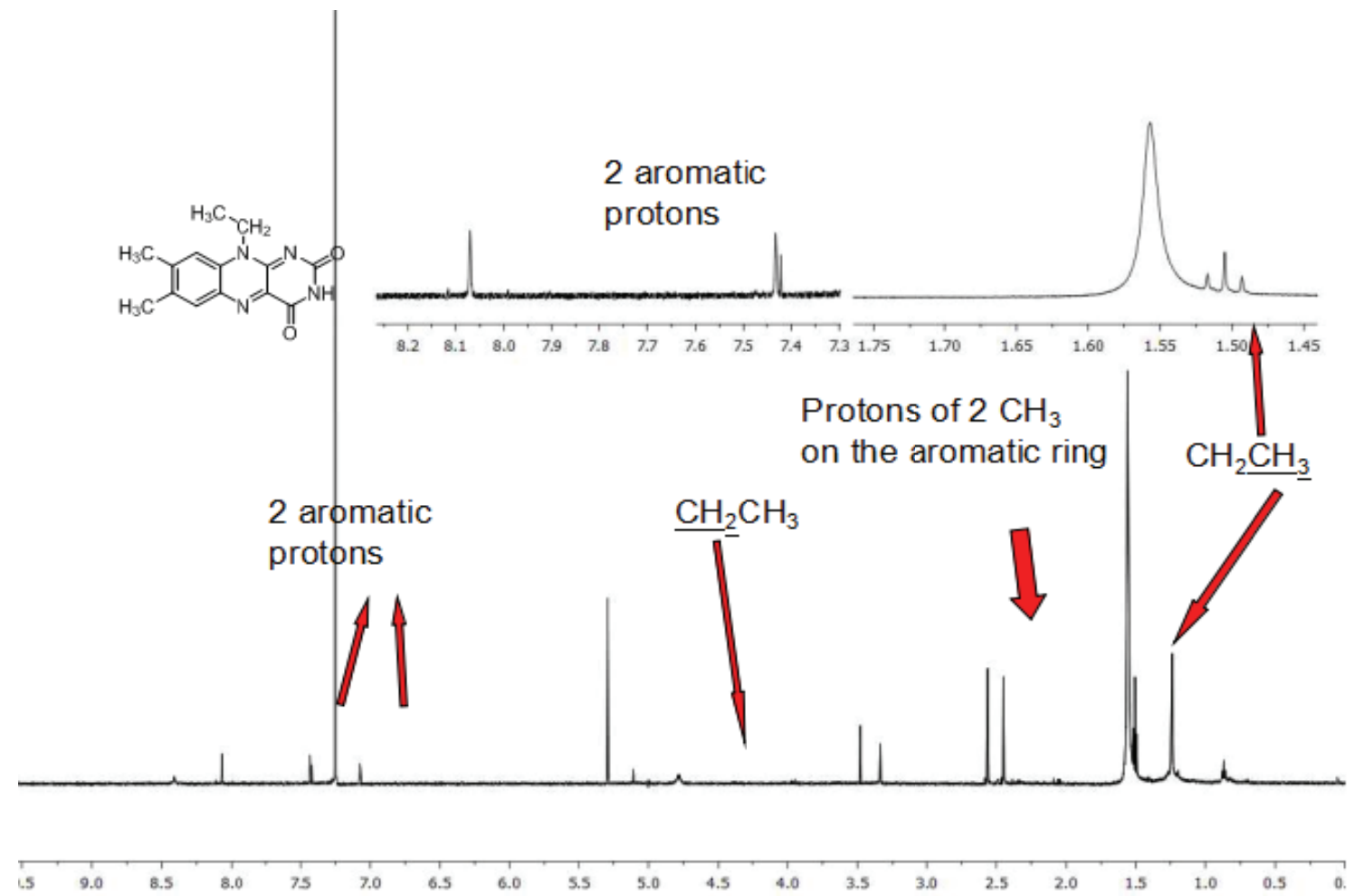

Fig. 4 HNMR for 10-ethyl flavin. 


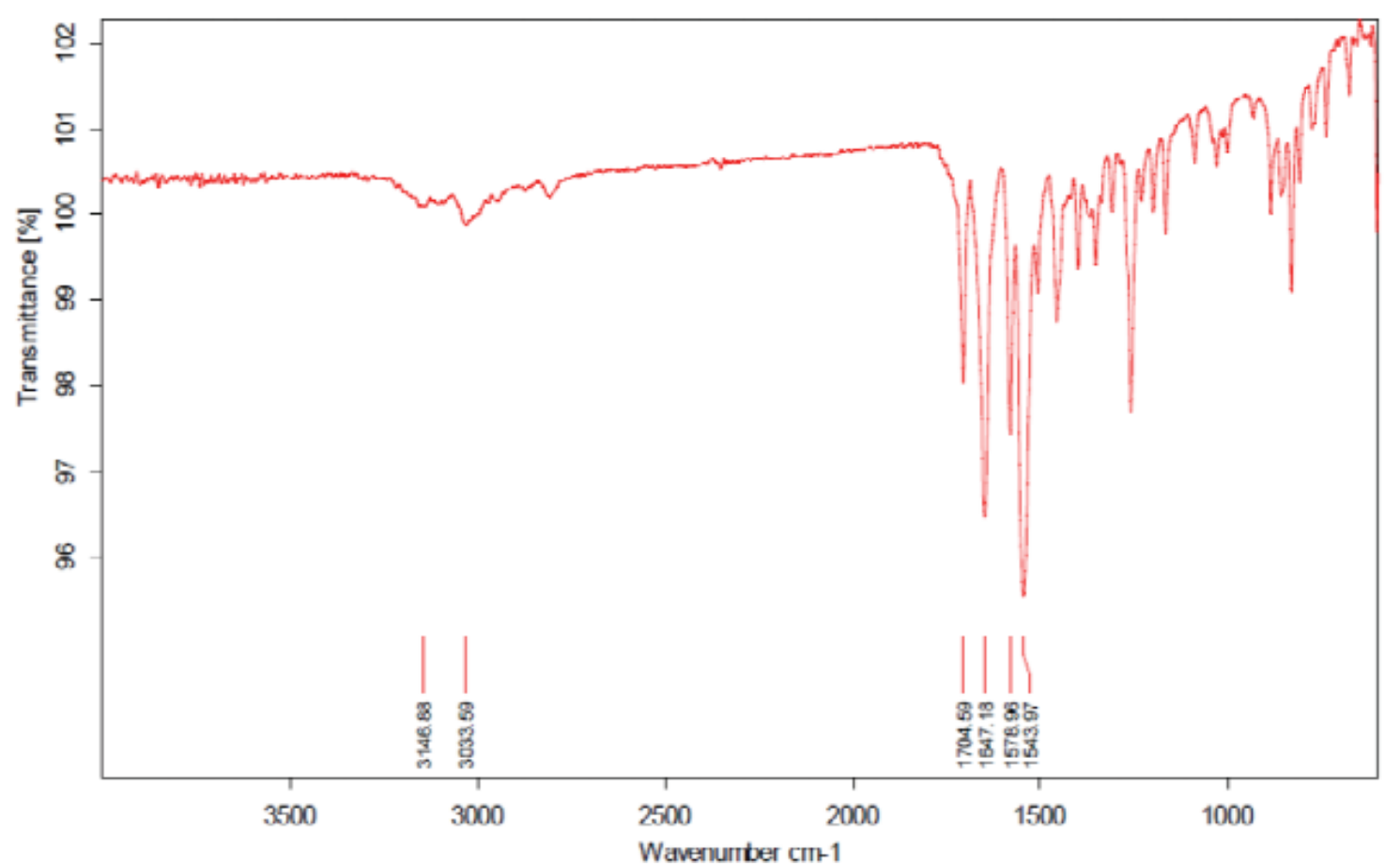

Fig. 5 IR spectrum for 10-ethyl flavin.

in hot water, and refrigerated for recyrsatlization to produce 10-ethyl falvin and analysis it for NMR spectra and IR spectra for 10-ethyl flavin shown as Fig. 4 and Fig. 5.

\subsection{The Degradation Experiment for Phenols}

In this study, two kinds of target organic synthesis product, 10-ethyl flavin and 5-ethyl riboflavin, are completed. The organic synthesis products of 10-ethyl flavin and 5-ethyl riboflavin are natural organic derivatives of riboflavin in animals and plants [5]. The riboflavin and its derivatives were studied by previous research on the photocatalytic degradation to various phenolic pollutants in water when exposed to natural light. They can produce photopigment and lumiflavin which have more photosensitive catalytic effect and then makes the phenolic pollutants much easier to decompose. [6] Therefore, two synthetic derivatives of riboflavin derivatives were used under natural light to catalyze the photocatalytic degradation of aqueous solutions containing a variety of phenolic pollutants.

In this experiment, the compounds that are used for this analysis were phenol, p-nitrophenol, 2,4-dichlorophenoxyacetic acid, 4-chlorophenol, 4-methoxyphenol, 4-chloro-2-methylphenoxyacetic acid and 2,4,5-trichlorophenoxyacetic acid. As a general method, photochemical reactions were conducted in a phosphate buffer $(1 \mathrm{~mL})$ containing $200 \mu \mathrm{M}$ phenol, $10 \mu \mathrm{M}$ flavin (10-ethyl flavin or 5-ethyl riboflavin) in quartz cuvettes under direct sunlight from $5 \mathrm{~min}$ to $2 \mathrm{~h}$. The reaction products were then analyzed on a C-18 reverse phase column with a mixture of acetonitrile in water (35\%) as eluent using a Shimadzu HPLC. In addition, the reaction products were extracted with diethyl ether and quantified using an Agilent GC mass spectrometer. The calibration curves and relative response factors were determined, and the compound signals were confirmed using the NIST database.

\section{Results}

The experimental results of riboflavin and its organic synthetic derivatives for degradation of 6 kinds of phenols inwater are shown in Table 3. It can be concluded from the table. In the concentration of $200 \mu \mathrm{M}$ phenol, $10 \mu \mathrm{M}$ flavin (10-ethyl flavin or 5-ethyl 
Table 3 The degradation rate for phenolic polutants using organics.

\begin{tabular}{lllllll}
\hline \multirow{2}{*}{\begin{tabular}{l} 
Organics used for $\begin{array}{c}\text { The degradation rate for phenolic pollutants } \\
\text { reaction }\end{array}$ \\
\cline { 2 - 7 }
\end{tabular}} & phenol & 4-chlorophenol & p-nitrophenol & 4-methoxyphenol & $\begin{array}{l}\text { 2,4-dichlorophenoxyace } \\
\text { ticacid }\end{array}$ & $\begin{array}{c}\text { 4-chloro-2-methylpheno } \\
\text { xyacetic acid }\end{array}$ \\
\hline riboflavin & $29.6 \%$ & $5.17 \%$ & $14.5 \%$ & 0 & 0 & 0 \\
10-ethyl flavin & $37.4 \%$ & $91.8 \%$ & $42.6 \%$ & $83.89 \%$ & $60.41 \%$ & $55.91 \%$ \\
5-ethylriboflavin & $17.8 \%$ & $28.3 \%$ & $12.0 \%$ & $40.41 \%$ & $18.47 \%$ & $37.42 \%$ \\
\hline
\end{tabular}

riboflavin), both flavin derivatives were successfully synthesized and characterized by NMR and mass spectrometry. A slightly modified method for 10-ethyl flavin was developed. Both HPLC and GC-MS data suggested that all phenols were degraded in the presence of either flavin at micromolar concentrations under direct sun light. A rapid breakdown of the phenols with $35-80 \%$ degradation over the course of 2 $\mathrm{h}$ was observed. The degradation efficiency was clearly dependent on the phenol type. The rank order for the degradation efficiency over $2 \mathrm{~h}$ was 4-chlorophenol and 4-methoxyphenol ( 80\%) > phenoxyacetic acids $(60-65 \%)>$ nitrophenol and phenol $(\sim 35 \%)$. For calibration curves of phenols established using GC mass spectrometry, our results showed a steady decrease in signals from $1 \mathrm{mM}$ to $200 \mu \mathrm{M}$ phenol in a linear fashion and then a rapid signal drop from $200 \mu \mathrm{M}$. The phenol signals at concentrations below $50 \mu \mathrm{M}$ became unidentifiable. Therefore, it can be concluded that two kinds of riboflavin synthesis derivatives under natural light have ideal effect on the degradation of para substituted phenolic pollutants in water, and riboflavin is not good due to the photolysis of itself under the light.

\section{References}

[1] Chen, J. 2006. "Horseradish Peroxidase Immobilized on Pillared Interlayered Clay and Its Application in the Treatment of Phenolic Wastewater". MSc thesis, HeFei University of Technology.

[2] Escalada, J. P., Pajares, A., Gianotti, J., et al. 2009. "Photosensitized Degradation in Water of the Phenolic Pesticides Bromoxynil and Dichlorophen in the Presence of Riboflavin, as a Model of Their Natural Photodecomposition in the Environment." Journal of Hazardous Materials 186 (1): 466-72.

[3] Reddy, R. B. Y. V. 2008. "Riboflavin Photosensitized Oxidation of Amino Acids." MSc thesis, The Ohio State University.

[4] Remucal, C. K., and Mcneill, K. 2011. "Photosensitized Amino Acid Degradation in the Presence of Riboflavin and Its Derivatives." Environmental Science \& Technology 45 (12): 5230-7.

[5] Yettella, R. R., and Min, D. B. 2010. "Effects of Trolox and Ascorbic Acid on the Riboflavin Photosensitised Oxidation of Aromatic Amino Acids." Food Chemistry 118 (1): 35-41.

[6] Zhang, T., Zhao, Q., Huang, H., et al. 1998. "Kinetic Study on the Removal of Toxic Phenol and Chlorophenol from Waste Water by Horseradish Peroxidase." Chinese Journal of Enviromentalence 37 (8): 1571-7. 